\title{
A Structural, Cognitive, and Behavioral Model for Error Analysis of Group B Streptococcus Prophylaxis in Pregnancy
}

\author{
Robert E. Murphy, MD ${ }^{1}$ Jane C. Ibekwe, RN, MS ${ }^{1}$ \\ ${ }^{1}$ School of Biomedical Informatics, University of Texas Health Science \\ Center at Houston (UT Health), Houston, Texas \\ 2 Division of Maternal Fetal Medicine, Department of Obstetrics, \\ Gynecology and Reproductive Sciences, UT Health-McGovern \\ Medical School, Houston, Texas
}

AJP Rep 2022;12:e17-e26.

\author{
Stella I. Ibekwe, RN, MS ${ }^{1}$ Jerrie S. Refuerzo, MD²
}

Address for correspondence Robert E. Murphy, MD, School of Biomedical Informatics, University of Texas Health Science Center at Houston, 7000 Fannin Street, Suite E710.F, Houston, TX 77030 (e-mail: Robert.murphy@uth.tmc.edu).

\begin{abstract}
Keywords

- error management and prevention

- sociotechnical aspects of information technology

- cognition

- clinical practice guideline

- group B streptococcus

The objective of this study was to develop a structural-cognitive-behavioral model for error analysis of group B streptococcus (GBS) prophylaxis failure, classify delivery cases into this model, and examine compliance with treatment guidelines. A retrospective, cohort study was conducted of women with liveborn pregnancies greater than 24 weeks in April 2018 at a single hospital. We created a structural-cognitive-behavioral model of five assessments for adherence to GBS prophylaxis guidelines and then classified these into four distinct error stages. A descriptive analysis was performed to determine if the pregnancy had a perfect process, a GBS prophylaxis failure, or a fortuitous outcome. There were 313 women who met the study criteria. The rate of GBS positive was $12.8 \%$, negative $37.4 \%$, and unknown $49.8 \%$. The most common errors were cognitive perception errors related to incorrectly documenting GBS status, $57.7 \%(N=79)$. Of these errors, $15.2 \%(N=12)$ led to GBS prophylaxis failure. Perfect outcomes occurred in $62.7 \%(N=196)$ women, GBS prophylaxis failure occurred in $13.7 \%(N=43)$, and fortuitous outcomes occurred in $23.6 \%(N=74)$. In our study, we were able to identify structural, cognitive, and behavioral errors that contribute to GBS prophylaxis failures. In other cases, these errors may contribute to fortuitous outcomes.
\end{abstract}

Group B streptococcus (GBS) is a Gram-positive bacterium that may colonize in women during pregnancy and may cause early- or late-onset sepsis in neonates. It remains the primary organism causing early-onset neonatal sepsis and is the leading infectious cause of newborn morbidity and mortality in the United States. ${ }^{1}$ As a result of widespread adoption of the Centers for Disease Control (CDC) treatment and prevention guidelines, there has been a significant decline in the incidence of GBS infected infants over the past 15 years. According to the $C D C$, the rate of early-onset infections has declined from 1.7 cases per 1,000 live births in 1993 , to 0.22 cases per 1,000 live births in $2016 .^{2}$ At the time of our study in 2018, adherence to the CDC guidelines of universal screening between 35 and 37 weeks of gestation for maternal GBS colonization, and treatment with intrapartum antibiotic prophylaxis has produced a notable received

June 22, 2021

accepted after revision

October 8, 2021
DOI https://doi.org/

$10.1055 / \mathrm{s}-0041-1742235$. ISSN 2157-6998.

\footnotetext{
(C) 2022. The Author(s).

This is an open access article published by Thieme under the terms of the Creative Commons Attribution-NonDerivative-NonCommercial-License, permitting copying and reproduction so long as the original work is given appropriate credit. Contents may not be used for commercial purposes, or adapted, remixed, transformed or built upon. (https://creativecommons.org/ licenses/by-nc-nd/4.0/)

Thieme Medical Publishers, Inc., 333 Seventh Avenue, 18th Floor New York, NY 10001, USA
} 
decrease in the burden of early-onset GBS infections in newborns. ${ }^{1}$ However, one of the largest epidemiologic studies on early-onset neonatal sepsis reported that only $76 \%$ of GBS colonized mothers received antibiotic prophylaxis. $^{3}$ A GBS positive pregnant woman that receives GBS prophylaxis per $C D C$ guidelines only has a 0.25 in 1,000 chance of delivering a GBS infected infant and a 5 in 1,000 chance if prophylaxis is not received per CDC guidelines. ${ }^{4}$ Hence, untreated GBS positive pregnant women are 20 times more likely than treated pregnant women to transmit the GBS bacteria to their infants. In addition to the challenges of administering antibiotics to at-risk women to prevent early-onset neonatal sepsis, concerns have been raised that overtreatment may lead to antibiotic resistance, or an increase in late-onset neonatal infections. ${ }^{5,6}$ While these studies identified several variables including individual clinician errors correlated to GBS prophylaxis compliance and noncompliance, there has not yet been a detailed analysis of the structural, cognitive, and behavioral factors that lead to noncompliance.

Compliance to guidelines can be evaluated into components that identify exact errors using a structural, cognitive, and behavioral analysis. Structural components influencing compliance include the role of interoperability and usability of electronic health records (EHRs). The final decision to order prophylactic antibiotics for GBS occurs in the hospital on the labor and delivery unit, but in many environments the screening for GBS has occurred in an outpatient clinic which may be using a different EHR. For many health systems, data availability becomes a major issue and a system contributor to possible compliance failure. Next, cognitive factors can be best understood by examining the work of researchers on situational awareness. Endsley, working initially in aviation environments, was the first to describe situational awareness as "a person's perception of the elements in the environment within a volume of time and space, the comprehension of their meaning, and the projection of their status in the near future."7 In recent years, situational awareness analysis has been applied to the health care environment, including anesthesia, ${ }^{8}$ pediatrics, ${ }^{9}$ critical care, ${ }^{8,10}$ labor and delivery units, ${ }^{11}$ and primary care settings. ${ }^{12}$ As related to GBS prophylaxis, situational awareness involves the correct perception of a situation (the patient's GBS status), comprehension of the meaning of the situation (whether this status and other risk factors indicate the need for GBS prophylaxis), and, finally, a projection of the implications of the situation and what actions may be necessary (ordering the antibiotic to reduce risk of early-onset neonatal sepsis). Finally, behavioral components focus on the aspect of administering the correct antibiotics and correct dose on time. Poon et al reported in a pre-/poststudy of barcoded medication administration that timing errors occurred in $16.7 \%$ of all medications administered before barcoded medications were implemented and $12.2 \%$ after implementation. ${ }^{13}$ Timing of antibiotics is particularly challenging in patients in active labor, who may have altered pharmacokinetic profiles due to pregnancy and labor may proceed with unanticipated urgency.
GBS prophylaxis compliance is a complex, multistep process that has structural, cognitive, and behavioral factors that inhibit guideline adherence. We hypothesized that lack of accessibility to previously obtained GBS laboratory results (i.e., data accessibility error) would be the most likely cause of GBS prophylaxis failure. Therefore, the objective of this study was to develop a structural, cognitive, and behavioral model for error analysis of GBS prophylaxis failure. We also sought to classify delivery cases into this model and examine compliance and failures with CDC treatment guidelines.

\section{Methods}

A cross-sectional, cohort study was conducted at a single tertiary care institution during a 1-month period. We included pregnant women from departmental electronic birth logs who presented for delivery at gestational ages greater than 24 weeks. Women with stillbirths were excluded.

Our focus hospital was part of a larger health care system. All facilities in the system used the same EHR and data obtained at any site was visible to all other sites. In the outpatient ambulatory setting, patients may have obtained their prenatal testing from various outpatient laboratories, including the hospital system, in which case the results from the hospital outpatient laboratory were visible on labor and delivery. In some cases, other outpatient laboratory results could have been sent electronically to the ambulatory EHR, and some reports were sent by facsimile machine and/or scanned into the ambulatory EHR. The ambulatory EHR was available for remote viewing from the hospital labor and delivery unit. The institutions each participated in the regional health information exchange (HIE), and the HIE data are available to clinicians in both institutions; however, microbiology results are not transmitted within the HIE.

We conducted an extensive chart review of the EHR including both the outpatient clinic and inpatient hospital settings. Both settings used EHR systems with a dedicated maternity component, but each from a different vendor. We collected both structured and unstructured EHR data. Structured data included clinical information that could be extracted within standard department reports and laboratory results. Unstructured data included manual review of clinical documents, such as clinic notes, scanned documents, emergency department provider notes, admission history and physicals, progress notes, and selected nursing assessments.

We determined the pregnancy GBS status from (1) a rectal-vaginal culture for GBS within 5 weeks prior to delivery; (2) a positive urine culture of GBS with greater than 10,000 colony-forming units per milliliter during the pregnancy; or (3) nursing documentation of "Transcribed GBS Status" based on a patient showing a hard copy result (e.g., prenatal laboratory summaries) in their possession during labor and delivery triage. This patient-supplied information is not retained by the hospital, and could not be analyzed as a possible source of transcription error.

Antibiotic orders and medication administration events were reviewed for the correct antibiotic, correct dose, and 
correct frequency in compliance with the CDC guidelines. Medication administration timeliness was defined as administration within 1 hour before or after scheduled time according to hospital policy. Finally, maternal demographics and pregnancy outcomes were collected. Our structural-cognitive-behavioral model ( - Fig. 1) includes five components for adherence to GBS prophylaxis guidelines: data existence, data accessibility, correct documentation, appropriate decision-making, and order followed (-Table 1). These five components are then classified into four distinct error stages: stage 1-data accessibility error, stage 2-perception error, stage 3-comprehension/decision error, or stage 4behavioral error ( $\mathbf{- T a b l e ~} \mathbf{2}$ ).

The structural component of the model included whether or not a GBS vaginal/rectal culture result and/or a positive urine culture of GBS during the current pregnancy were available in the respective EHRs ("data exists"). Next, we assessed whether these results were available within the hospital EHR ("data is accessible"). If data existed in the clinic EHR and was not visible in the hospital EHR, this was considered a stage 1 (data accessibility) error.

The cognitive component of the model focused on two aspects considered to be associated with situational awareness. First, the physician's perception of the results and comprehension of the significance of the patient's GBS status by ordering or not ordering antibiotics. We used documentation of GBS status in the admission and progress notes as a proxy for perception and assessed the accuracy and consistency of this documentation ("documented correctly"). While we acknowledge that cognitive perception may certainly occur in the absence of documentation, documentation is the only way in which we can confirm the clinician's recognition. A failure of correct documentation is therefore a stage 2 (perception) error. Second, the assessment of whether or not antibiotics were ordered correctly ("appropriate decision") was made in regard to the retrospective determination of the patient's GBS status and indication for GBS prophylaxis. We do split stage 3 (comprehension/decision) errors into subclass (1) omission, defined as GBS prophylaxis is indicated, but an antibiotic was not ordered; and (2) commission defined as GBS prophylaxis not indicated, but an antibiotic was ordered.

The behavior component of the model is whether the antibiotic orders were followed correctly ("orders followed") including the correct dose, correct frequency, and medication administration timeliness. A failure to correctly follow the order is considered a stage 4 (behavioral) error.

We completed a thorough analysis of each case to determine if the pregnancy had a perfect outcome, GBS prophylaxis failure, or a fortuitous outcome in terms of adherence to

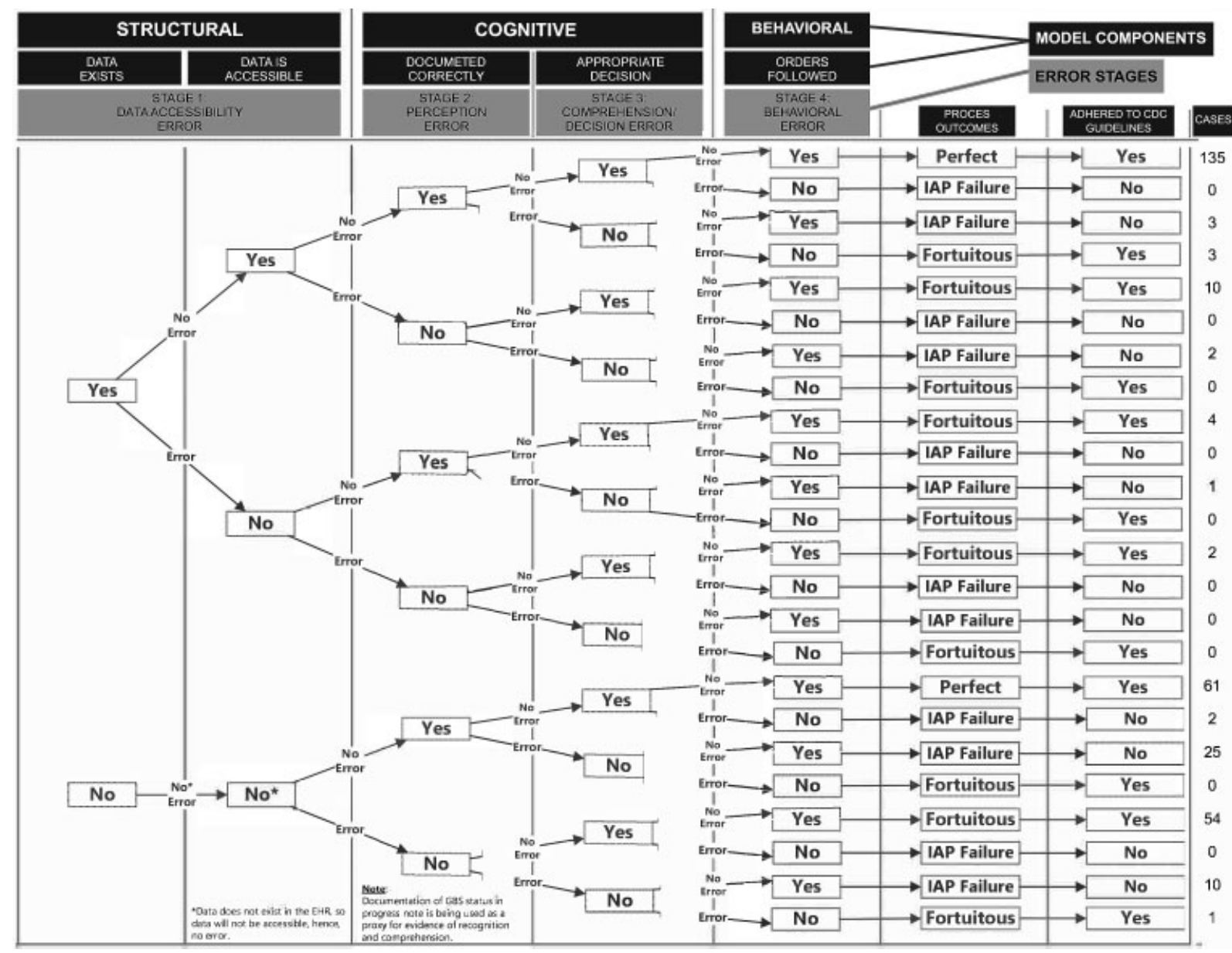

Fig. 1 Structural, cognitive, and behavioral model for error analysis of intrapartum antibiotic prophylaxis (IAP) failure in group B streptococcus (GBS) prophylaxis guideline compliance. 
Table 1 Structural, cognitive, and behavioral components defining the criteria of GBS prophylaxis adherence

\begin{tabular}{|c|c|c|}
\hline & & Criteria \\
\hline \multirow[t]{2}{*}{ Structural components } & $\begin{array}{l}\text { Did the data exist in } \\
\text { an EHR? }\end{array}$ & $\begin{array}{l}\text { Yes, if GBS vaginal/rectal screen, urine culture with GBS or transcribed GBS } \\
\text { result is present in clinic and/or hospital EHR prior to delivery date/time; } \\
\text { or if present GBS result is expired (i.e., > } 5 \text { weeks old-it becomes invalid, } \\
\text { but data still exists) } \\
\text { No, if no results available }\end{array}$ \\
\hline & $\begin{array}{l}\text { Was data accessible } \\
\text { in the hospital EHR? }\end{array}$ & $\begin{array}{l}\text { Yes, if GBS vaginal/rectal screen, urine culture with GBS or transcribed GBS } \\
\text { result is present in the hospital EHR prior to delivery date/time; or if } \\
\text { present GBS result is expired (i.e., }>5 \text { weeks old-it becomes invalid, but } \\
\text { data are still accessible) } \\
\text { No, if data did not exist, or data existed in clinic EHR, but not visible in } \\
\text { hospital EHR }\end{array}$ \\
\hline \multirow[t]{2}{*}{ Cognitive components } & $\begin{array}{l}\text { Was pregnancy GBS } \\
\text { status documented } \\
\text { correctly? }\end{array}$ & $\begin{array}{l}\text { Yes, if pregnancy GBS status is accurately and consistently documented by } \\
\text { physician in hospital EHR note } \\
\text { No, if pregnancy GBS status is inaccurately documented by physician in } \\
\text { hospital EHR note }\end{array}$ \\
\hline & $\begin{array}{l}\text { Was the decision to } \\
\text { order or not order } \\
\text { antibiotics } \\
\text { appropriate? }\end{array}$ & $\begin{array}{l}\text { Yes, if patient meets CDC criteria to treat (antibiotics indicated), and } \\
\text { antibiotics were ordered by physician; or if patient does not meet CDC } \\
\text { criteria to treat (antibiotics not indicated), and antibiotics were not } \\
\text { ordered by physician } \\
\text { No, if patient meets CDC criteria to treat (antibiotics indicated), and } \\
\text { antibiotics were not ordered by physician; or if patient does not meet CDC } \\
\text { criteria to treat (antibiotics not indicated), and antibiotics were ordered by } \\
\text { physician }\end{array}$ \\
\hline Behavioral component & $\begin{array}{l}\text { Was the order fol- } \\
\text { lowed by the nurse? }\end{array}$ & $\begin{array}{l}\text { Yes, if antibiotics were ordered by physician and the correct dose was } \\
\text { administered by nurse on time; or if antibiotics were not ordered by } \\
\text { physician and not administered by nurse } \\
\text { No, if antibiotics were ordered by physician and the correct dose was not } \\
\text { administered on time by nurse, or correct dose was administered late ( }> \\
1 \text { hour); or if antibiotics were not ordered by physician, but still admin- } \\
\text { istered by nurse }\end{array}$ \\
\hline
\end{tabular}

Abbreviations: CDC, Centers for Disease Control; EHR, electronic health record; GBS, group B streptococcus.

the care processes described in the CDC guidelines. A perfect outcome was defined as no error present and the correct outcome achieved. GBS prophylaxis failure was defined as at least one error present and correct outcome not achieved. A fortuitous outcome was defined as one or more errors present, but the correct outcome was still achieved.

\section{Results}

There were 324 women who delivered during the month of April 2018. There were 11 women excluded from our analysis due to: 1 had no delivery type entered in the electronic birth $\log , 2$ vaginal deliveries had no recorded gestational age, and

Table 2 Structural, cognitive, and behavioral stages of errors in GBS prophylaxis adherence

\begin{tabular}{|c|}
\hline Stage 1-Data accessibility error \\
\hline $\begin{array}{l}\text { Data exists in an EHR (clinic and/or hospital), but is not accessible by physician in the EHR used in the hospital's labor } \\
\text { and delivery unit }\end{array}$ \\
\hline Stage 2-Perception error \\
\hline $\begin{array}{l}\text { Physician failed to accurately and consistently document the correct pregnancy GBS status in progress notes. } \\
\text { Documentation of pregnancy GBS status in progress notes is being used as a proxy for evidence of perception }\end{array}$ \\
\hline Stage 3-Comprehension/Decision error \\
\hline $\begin{array}{l}\text { a. IAP is indicated, but physician failed to order appropriate antibiotics (omission error) } \\
\text { b. IAP is not indicated, but physician ordered appropriate antibiotics (commission error) }\end{array}$ \\
\hline Stage 4-Behavioral error \\
\hline $\begin{array}{l}\text { a. Physician ordered antibiotics, but antibiotics were not administered by nurse (no treatment) } \\
\text { b. Physician ordered antibiotics, but loading dose of antibiotics were not administrated (dosage undertreatment), } \\
\text { or antibiotics were not administrated on time (timing undertreatment) }\end{array}$ \\
\hline
\end{tabular}

Abbreviations: EHR, electronic health record; GBS, group B streptococcus; IAP, intrapartum antibiotic prophylaxis. 
8 cesarean deliveries had no recorded status of amniotic membrane rupture. The remaining 313 women were included in our cohort for analysis. Our maternal population included 33.9\% $(N=106)$ African American, $89.1 \%$ $(N=279)$ maternal age between 19 and 39 years old, 30.7\% $(N=96)$ were nulliparous, and cesarean section rate was 43.1\% $(N=135)$ and 26.5\% $(N=83)$, delivered less than 37 weeks' gestation as shown in - Table 3. Overall, positive GBS status was identified in $12.8 \%(N=40)$ of all patients, and $25.5 \%$ of patients tested, and negative in $37.4 \%(N=117)$, $74.5 \%$ of patients tested. Unknown GBS status was identified in $49.8 \%(N=156)$.

-Fig. 2 illustrates the assessment of cases regarding the structural, cognitive, or behavioral components of adherence to GBS prophylaxis guidelines. Structurally, $51.1 \%(N=160)$

Table 3 Maternal demographics and characteristics

\begin{tabular}{|c|c|c|}
\hline \multicolumn{3}{|l|}{ Characteristics } \\
\hline \multirow[t]{6}{*}{ Maternal age $(\mathrm{Y})^{\mathrm{a}}$} & $15-19$ & $25(8)$ \\
\hline & $20-24$ & $64(20.4)$ \\
\hline & $25-29$ & $82(26.2)$ \\
\hline & $30-34$ & $88(28.1)$ \\
\hline & $35-39$ & $45(14.4)$ \\
\hline & $\geq 40$ & $9(2.9)$ \\
\hline \multirow[t]{6}{*}{ Maternal race ${ }^{a}$} & White (Caucasian) & $63(20.1)$ \\
\hline & $\begin{array}{l}\text { Black } \\
\text { (African American) }\end{array}$ & 106 (33.9) \\
\hline & Asian & $14(4.5)$ \\
\hline & Native American & $2(0.6)$ \\
\hline & Other & $122(39)$ \\
\hline & Unknown & $6(1.9)$ \\
\hline \multirow[t]{3}{*}{ Parity $^{a}$} & None & $0(0)$ \\
\hline & 1 & $96(30.7)$ \\
\hline & $\geq 2$ & $217(69.3)$ \\
\hline \multirow[t]{4}{*}{ Previous live birth ${ }^{a}$} & None & $17(5.4)$ \\
\hline & 1 & $109(34.8)$ \\
\hline & $\geq 2$ & $181(57.8)$ \\
\hline & Unknown & $6(1.9)$ \\
\hline \multirow[t]{2}{*}{ Delivery type $^{a}$} & Vaginal & $178(56.9)$ \\
\hline & Cesarean & $135(43.1)$ \\
\hline \multirow[t]{3}{*}{ Term, preterm status ${ }^{a}$} & Preterm $^{\mathrm{b}}$ & $83(26.5)$ \\
\hline & Term $^{c}$ & 225 (71.9) \\
\hline & Unknown & $5(1.6)$ \\
\hline \multirow[t]{3}{*}{ GBS status } & Positive & $40(12.8)$ \\
\hline & Negative & $117(37.4)$ \\
\hline & Unknown & $156(48.8)$ \\
\hline
\end{tabular}

Abbreviation: GBS, group B streptococcus.

${ }^{a} n(\%)$. Percentages are calculated based on total maternal patients analyzed (313).

'Indicates delivery at $<37$ weeks' gestation.

'Indicates delivery at $\geq 37$ weeks' gestation. had a GBS result that existed in either the clinic or hospital EHR. However, 7 women (2.2\%) did not have these results in the hospital EHR due to the laboratories existing in an external laboratory system that did not interface with the hospital's EHR (-Fig. 3$)$. Therefore, only $48.9 \%(N=153)$ had their GBS result accessible at the time of delivery.

Cognitively, $74.8 \%$ of women $(N=234)$ had their GBS status documented correctly and consistently in the physicians' notes within the hospital EHR (-Fig. 2). Although $85.6 \%$ of women $(N=268)$ had appropriate decision making, $14.4 \%(N=45)$ did not. Of these 45 cases, $33.3 \%(N=15)$ were errors by omission in which the physician did not order antibiotics when indicated, and $66.7 \%(N=30)$ were errors by commission in which antibiotics were ordered when not indicated (-Fig. 3).

Behaviorally, 98.1\% $(N=307)$ orders were followed correctly. There were six cases of behavioral errors in which the antibiotics were not given correctly. In one case, the correct loading dosage was not administered and in five cases at least one of the doses for the patient was administered late.

In total, 137 errors were found in 120 cases from a combination of structural, cognitive, or behavioral components of adherence; therefore, errors occurred in $38.3 \%$ of the 313 patients. In 17 cases (12.4\%), there was more than one error.

-Figs. 3 and 4 cover the frequency of structural, cognitive, and behavioral errors and their frequency in leading to GBS failure, respectively. Stage 1 (data accessibility) errors occurred in $2.2 \%(N=7)$ of patients and only $1(14.2 \%)$ of these contributed to a GBS prophylaxis failure. Overall, perception errors (stage 2) related to correctly and consistently documenting GBS status was the most common error in all patients, accounting for $57.7 \%(N=79)$ of total errors. Of these 79 perception errors, $15.2 \%(N=12)$ resulted in GBS prophylaxis failure. Comprehension and decision errors (stage 3$)$ accounted for $32.8 \%(N=45 / 137)$ of total errors, and $91.1 \%(N=41)$ resulted in GBS prophylaxis failures. Behavioral errors (stage 4$)$ were found in $1.9 \%(N=6)$ of total errors and of these $33.3 \%(N=2)$ contributed to GBS prophylaxis failure. Overall with 137 total errors, $40.9 \%(N=56)$ of these were associated with GBS prophylaxis failure.

Thirteen patients had perception errors (incorrect documentation) that were coupled with a comprehension/decision error. Eight of these cases were documented as positive GBS status and five were documented as negative GBS status; however, with no data available, these were ruled as unknown status. Twelve of the 13 cases had antibiotics when not indicated. The remaining case was the only omission error. The patient was documented as negative GBS status but had an intrapartum temperature greater than $100.4^{\circ} \mathrm{F}$ and should have received prophylaxis.

In our study, perfect outcomes occurred in $62.7 \%$ $(N=196)$ women, GBS prophylaxis failure occurred in $13.7 \%(N=43)$, and fortuitous outcomes in $23.6 \%(N=74)$ as shown in - Fig. 5. GBS prophylaxis failure is the most concerning. Of the GBS prophylaxis failures, $5.4 \%(N=17)$ of cases did not receive antibiotics when indicated. Cognitive 


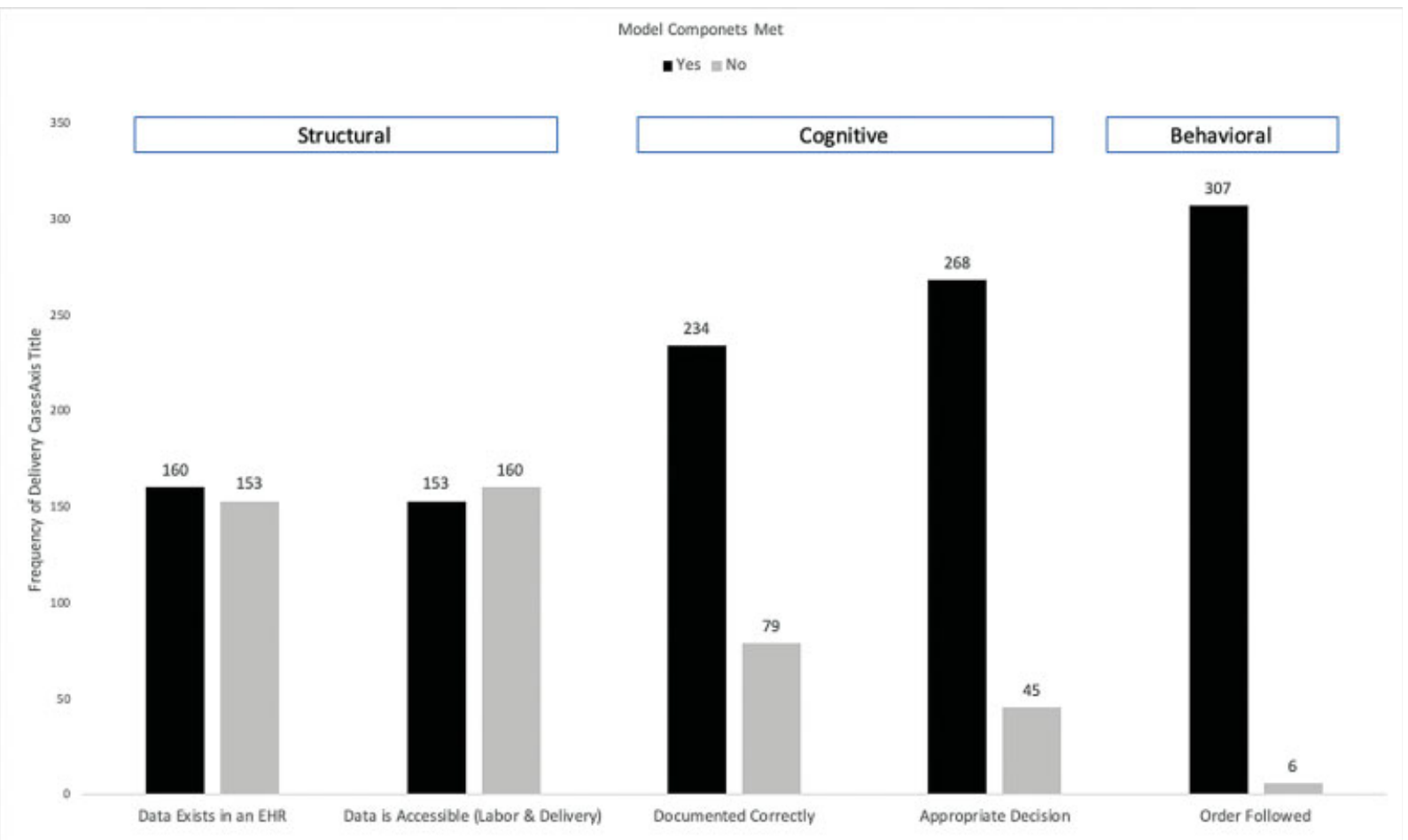

Fig. 2 Frequency of structural, cognitive, and behavioral components met for analysis of adherence to group B streptococcus (GBS) prophylaxis guidelines.

errors in comprehension and decision making occurred in 15 of the 17 cases (88.2\%), while behavioral errors accounted for the remaining 2 cases (11.8\%). Most of these cases $(82.2 \% ; N=37 / 45)$ involved an unknown GBS status and additional risk factors present. One of these cases involved a patient who had a negative GBS rectal-vaginal screen greater than 5 weeks prior to delivery. The patient therefore reverted to "unknown" status and she had a fever prior to delivery, which qualifies it as a GBS prophylaxis failure. Finally, $8.3 \%(N=26)$ of GBS prophylaxis failures were due to overtreatment and given antibiotics when not indicated.

Fortuitous outcomes are unique cases and occurred in 74 women. Despite an identified error, the majority of women $93.2 \%(N=69)$ resulted in no antibiotics given when not indicated. There were five cases in which antibiotics were ordered and administered when indicated despite an error, all of them due to documentation. One

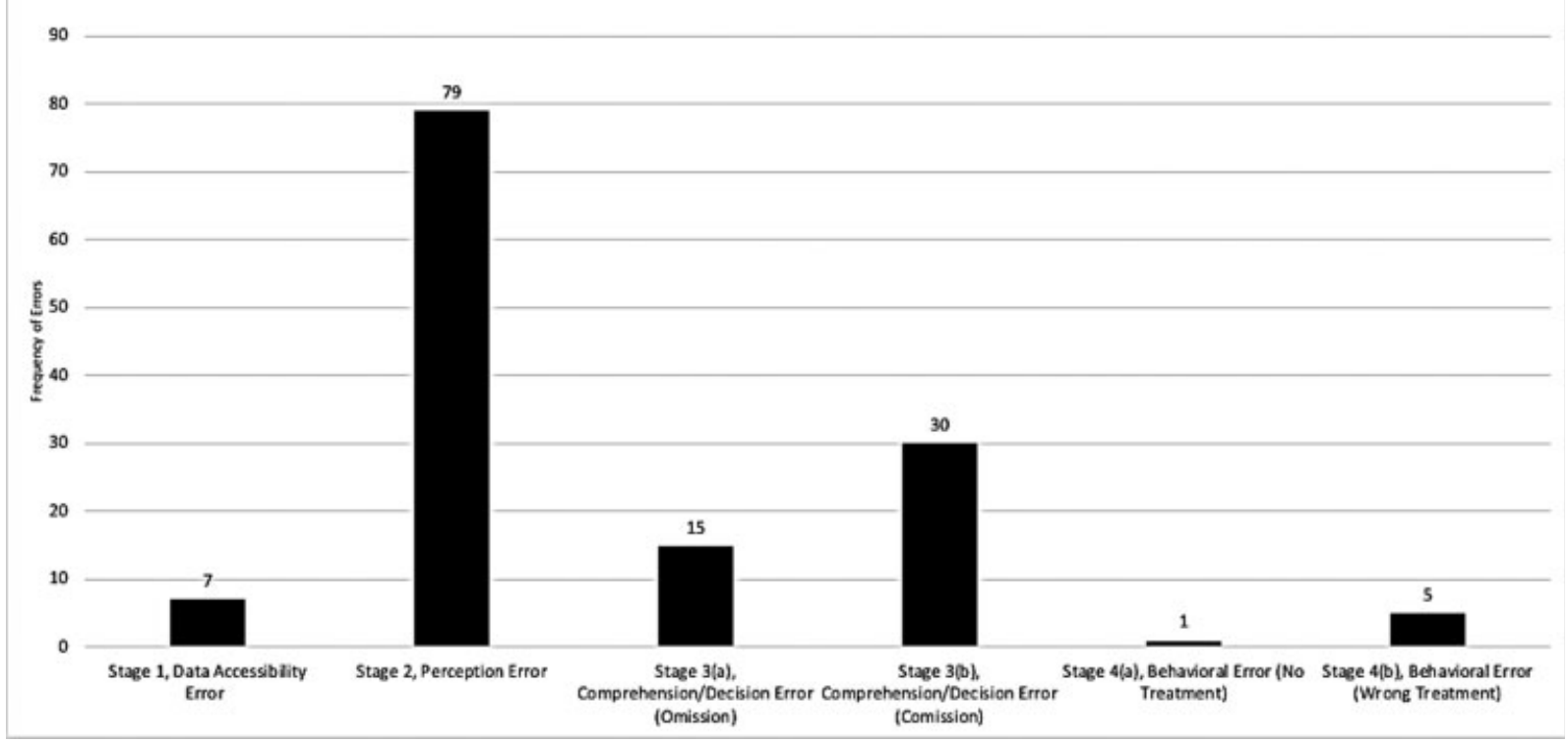

Fig. 3 Frequency of structural, cognitive, and behavioral errors for analysis of adherence to group B streptococcus (GBS) prophylaxis guidelines. 


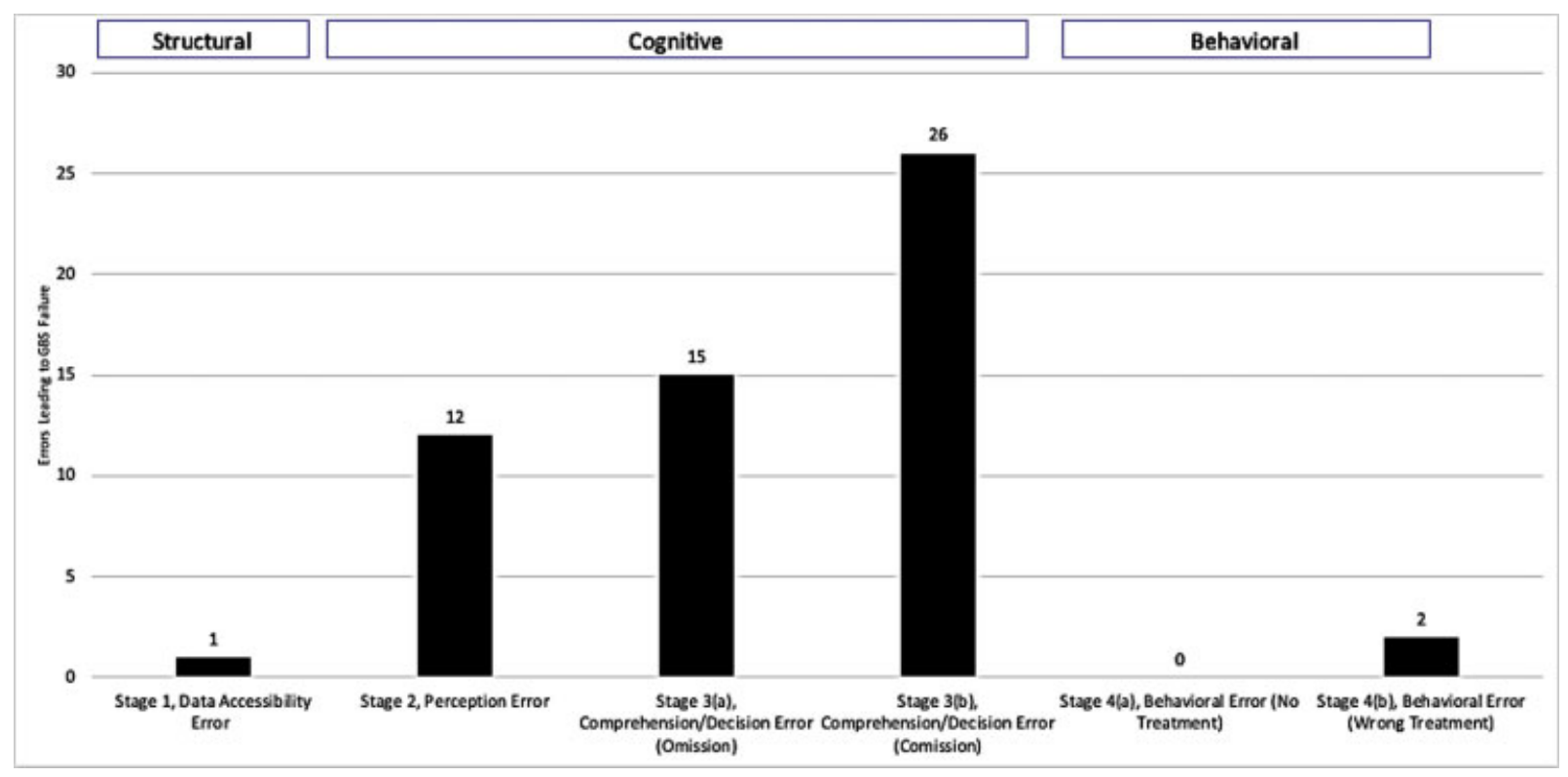

Fig. 4 Frequency of structural, cognitive, and behavioral errors that are associated with group B streptococcus (GBS) prophylaxis failures.

case occurred in a woman with GBS positive result who was documented to be unknown. Three cases who were in reality unknown status but were documented as positive GBS status with no supporting laboratory results or evidence. However, GBS prophylaxis became indicated due to preterm gestational age. The fifth fortuitous case occurred in a woman who had a positive urine culture at an external clinic 21 weeks prior to delivery. This result was in a scanned document in the ambulatory clinic EHR and not visible in the hospital EHR. The nurse transcribed GBS status as unknown, yet the physician did correctly document the positive status and treated

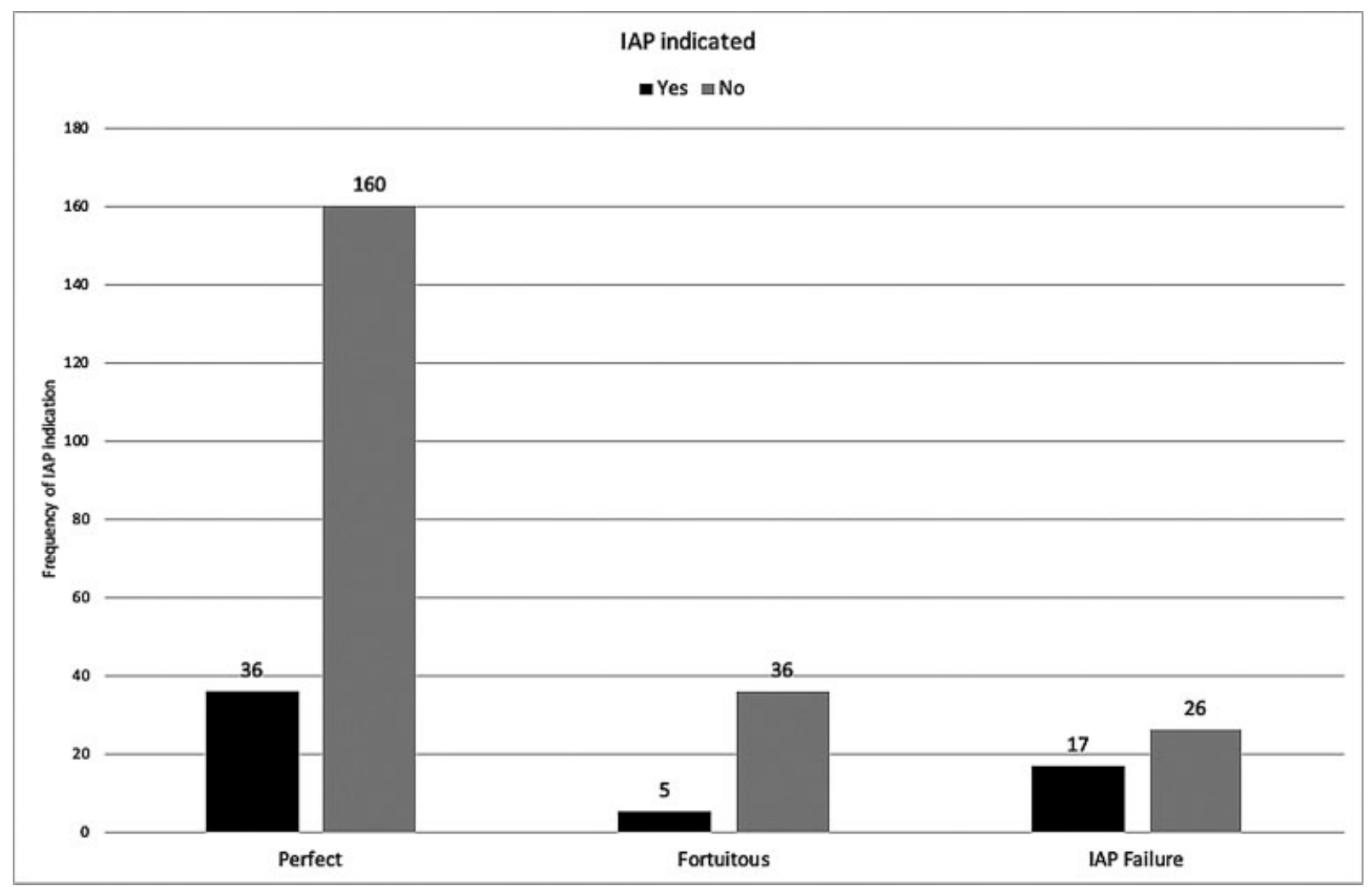

Fig. 5 Frequency of outcomes related to intrapartum antibiotic prophylaxis (IAP) indication. A "perfect" outcome is compliance with the Centers for Disease Control (CDC) group B streptococcus (GBS) prophylaxis guidelines without error. A "fortuitous" outcome is compliance with the guidelines, yet one or more errors were present. IAP failure occurs with failure to comply with guidelines with one or more errors present. 
the patient appropriately. It is not known whether the physician logged into the ambulatory EHR, or if the patient verbally informed the physician of the result. However, this is still a structural, data accessibility error, yet a fortuitous outcome.

\section{Discussion}

Prophylaxis for GBS is a complex process. We were able to identify structural, cognitive, and behavioral errors that may contribute to failures in many cases, but in other cases, these errors may create fortuitous outcomes. Although we had hypothesized that structural components such as data accessibility to GBS results would lead to the most prophylactic failures, this was not found. In our study, GBS results were accessible within the hospital in approximately half the cases but contributed to less than $2 \%$ of GBS prophylactic failures. In contrast, comprehension and decision-making cognitive errors occurred in 45 cases (14.2\%) and led to over $90 \%$ of GBS prophylactic failures. Finally, there was only a $2 \%$ rate of behavioral errors that contributed to two cases of GBS prophylaxis failure due to antibiotic undertreatment.

While the incidence of early-onset neonatal sepsis has declined since the introduction of prevention guidelines by the CDC in 2010, several studies have shown that we have not yet approached complete screening or prophylaxis for all appropriate patients., ${ }^{3,8}$ Our study had a lower incidence of a perfect process of $62.6 \%$ compared with $76 \%$ previously reported. ${ }^{3}$ Bianco et al recently published a retrospective study characterizing the appropriateness of GBS prophylaxis in four hospitals in Italy. ${ }^{14}$ They reported 91.1\% of GBS prophylaxis administration in patients for whom prophylaxis was indicated, but also noted that only $36.3 \%$ of pregnant women had what they called "totally appropriate" prophylaxis, in regards to correct drug choice, route of administration, dosage regimen, and timing. Verani et al evaluated cases of early-onset GBS disease and determined that these cases had implementation errors in $57.9 \%{ }^{15}$

GBS prophylactic failures with overtreatment increases concern for antibiotic resistance and development of lateonset neonatal sepsis. An epidemiologic study of GBS isolates between 1999 and 2005 showed 32\% resistant to clindamycin and $15 \%$ resistant to erythromycin. ${ }^{5}$ In a small study from Utah, Glasgow et al reported an association of intrapartum antibiotic administration to an increased risk of late-onset (7-90 days) serious bacterial illness in neonates. 6

Our study does have limitations. We clearly had a high rate of patients with unknown GBS status, including preterm deliveries who would not have had GBS screening. We did not assess specific aspects related to GBS screening in this error analysis, as our focus was the labor and delivery unit itself. GBS screening is clearly an important component of overall GBS prevention, but this is a separate multifactorial process that requires its own analysis (e.g., patient scheduling, patient appointment compliance). Also, due to the complexity of individual patient care environments, our results may not be generalizable to other institutions; for instance, organizations which have a single EHR for the inpatient and outpatient settings, or on the other extreme, hospitals that have a higher proportion of mothers without prenatal care. Other limitations of our study are a small sample size, lack of access to all clinical results (e.g., free standing emergency departments or urgent care clinics), and our use of documentation to infer physician perception of available clinical data. Finally, for several of the cases we categorized as a comprehension/decision error, we must also acknowledge that EHR usability may be a contributing factor that we were unable to establish based on available data. During the period of analysis, we discovered that the default reference range for the microbiology results was set for 2 weeks. While physicians could individually alter this display range, our experience leads us to believe that many users accept the default and do not change it. The implication here could be significant in that 195 of 232 (84.1\%) GBS screens or urine culture results were resulted greater than 2 weeks prior to delivery. As a result of our analysis, the institution has changed the default display range for microbiology results to 9 months.

At our institution, this study was conceived after an analysis of a single neonatal sepsis case. In this case, the mother had a positive urine culture for GBS which was not available to the treating physician in the labor and delivery suite. The urine was obtained at an external laboratory, recorded in the clinic notes, but not visible in the hospital EHR. Based on this, our initial hypothesis was that the primary source of GBS prophylaxis failure was the lack of interoperability with laboratory results between the institutions.

Adherence to GBS prophylaxis guidelines is not as simple as checking a GBS status and treating when indicated. We created our structural-cognitive-behavioral model based on five key components and an error in one (or more) of these steps can lead to four potential sources of failure. Our most frequent error was a stage 2 (perception) error with incorrectly documenting the patient's GBS status in 79 (25.2\%) cases. We acknowledge that perception is not exclusively captured with documentation; however, it is the only permanent indicator of the physician recognizing GBS status and communicating this perception to the medical record and to other clinicians on the care team. Physician may be aware of a patient's GBS without documenting it or use temporary documentation to record this status. While electronic tracking boards of key information are becoming more common, many labor and delivery units may still use whiteboards to indicate patient status. We maintain that a failure to accurately and consistently document a patient's GBS status in the medical record is an error. Stage 3 (comprehension/decision) errors are the most common error type leading to GBS prophylaxis failure. The majority of these cases occurred when the GBS status was unknown. Stage 4 (behavioral) errors are not common and include late administrations. 
Fortuitous outcomes occurred in approximately 1 in 5 women and are described in our population as "lucky cases" where although there were one or more errors, the intended outcome was still achieved. It remains uncertain how to approach these cases.

While data accessibility errors were not the primary error type we uncovered, we did have seven cases in which data existed but was not available to the treating physician. We believe this structural error could be addressed by adopting a national electronic standard for reporting microbiology results as exists for all other clinical laboratory results.

Unknown GBS status with risk factors was the most common status associated with GBS prophylaxis failure. These cases represent the most challenging component of situational awareness, as the risk factor status is often dynamic in a time-sensitive, task-saturated clinical environment. Identification of structural, cognitive, and behavioral errors contributing to nonadherence to national guidelines is a novel concept. This angle into the complex world of clinical care can provide opportunities to emphasize importance of documentation, use of risk-based antibiotic guidelines, and to promote teamwork among health care providers. In the field of perinatal medicine located on a fast-paced, high turnover hospital unit such as labor and delivery, where access to prenatal laboratories and executing treatment when indicated is vital to the health and safety of both mother and infant, delving into the structural-cognitive-behavioral model outlined in this study may provide solutions to many unanswered clinical questions.

Finally, in July 2019, the American College of Obstetricians and Gynecologists provided updates to the 2010 CDC guidelines of GBS prophylaxis. ${ }^{16}$ The most notable change was narrowing the timing of GBS screening. This could have a downstream effect on adherence to GBS prophylaxis. Even small changes in clinical guidelines can be disruptive and we anticipate that more failures may occur with this new update. For instance, narrowing the window for GBS screening could lead to more patients classified as "unknown" status, which was the biggest source of error in our study. Perhaps applying this structural, cognitive, and behavioral model could bring to light the implementation of guidelines into clinical care.

\section{Clinical Relevance Statement}

We created a model to analyze the structural, cognitive, and behavioral components as detected from electronic health records in the process of group $B$ prophylaxis in pregnant patients. While errors in data availability or documentation were common, comprehension/decision-making errors were most correlated to GBS prophylaxis. Improved techniques for error detection and mitigation will need to be developed.

\section{Protection of Human and Animal Subjects}

This study was performed in compliance with the World Medical Association Declaration of Helsinki on Ethical
Principles for Medical Research and approved by the University of Texas Health Science Center at Houston Institutional Review Board: Committee for the Protection of Human Subjects (HSC-17-0617).

Source of Financial Support

Departmental at UT Health.

\section{Conflict of Interest}

The authors report no conflict of interest.

\section{Acknowledgments}

The authors would like to thank Dr. Alex Nguyen for his early work on data collection methods during a student rotation; Dr. Amy Franklin for her guidance on situational awareness and cognitive decision-making; Tina Harbert for assistance in data collection, EHR usability, and data process analysis; Dr. John Riggs for clinical perspectives and EHR usability; and Dr. Dean Sittig, Dr. Susan Wootton, Dr. Barbara Stoll, and Dr. Carol Baker for research insights and feedback on our manuscript.

\section{References}

1 Verani JR, McGee L, Schrag SJDivision of Bacterial Diseases, National Center for Immunization and Respiratory Diseases, Centers for Disease Control and Prevention (CDC) Prevention of perinatal group B streptococcal disease-revised guidelines from CDC, 2010. MMWR Recomm Rep 2010;59(RR-10, No. RR-10):1-36

2 Group B strep (GBS) Clinical Overview. Centers for Disease Control and Prevention website. Accessed December 2, 2018 at: http://www.cdc.gov/groupbstrep/clinicians/clinical-overview. html

3 Stoll BJ, Hansen NI, Sánchez PJ, et al; Eunice Kennedy Shriver National Institute of Child Health and Human Development Neonatal Research Network. Early onset neonatal sepsis: the burden of group B Streptococcal and E. coli disease continues. Pediatrics 2011;127(05):817-826

4 Group B strep (GBS) Fast Facts. Centers for Disease Control and Prevention website. Accessed December 2, 2018 at: http://www. cdc.gov/groupbstrep/about/fast-facts.html

5 Phares CR, Lynfield R, Farley MM, et al; Active Bacterial Core surveillance/Emerging Infections Program Network. Epidemiology of invasive group B streptococcal disease in the United States, 1999-2005. JAMA 2008;299(17):2056-2065

6 Glasgow TS, Young PC, Wallin J, et al. Association of intrapartum antibiotic exposure and late-onset serious bacterial infections in infants. Pediatrics 2005;116(03):696-702

7 Endsley MR. Design and evaluation for situation awareness enhancement. Proc Hum Factors Soc Annu Meet 1988;32(02): 97-101

8 Schulz CM, Krautheim V, Hackemann A, Kreuzer M, Kochs EF, Wagner KJ. Situation awareness errors in anesthesia and critical care in 200 cases of a critical incident reporting system. BMC Anesthesiol 2016;16:4

9 Brady PW, Muething S, Kotagal U, et al. Improving situation awareness to reduce unrecognized clinical deterioration and serious safety events. Pediatrics 2013;131(01):e298-e308

10 Rodriguez AC, Lee DA Jr, Makic MBF. Situational awareness in critical care: an aviation approach to reduce error. J Perianesth Nurs 2017;32(06):650-652

11 Edozien LC. Situational awareness and its application in the delivery suite. Obstet Gynecol 2015;125(01):65-69 
12 Singh H, Giardina TD, Petersen LA, et al. Exploring situational awareness in diagnostic errors in primary care. BMJ Qual Saf 2012;21(01):30-38

13 Poon EG, Keohane CA, Yoon CS, et al. Effect of bar-code technology on the safety of medication administration. N Engl J Med 2010; 362(18):1698-1707

14 Bianco A, Larosa E, Pileggi C, Pavia MCollaborative Working Group. Appropriateness of intrapartum antibiotic prophylaxis to prevent neonatal group B streptococcus disease. PLoS One 2016;11(11):e0166179

15 Verani JR, Spina NL, Lynfield R, et al. Early-onset group B streptococcal disease in the United States: potential for further reduction. Obstet Gynecol 2014;123(04):828-837

16 Prevention of Group B Streptococcal Early-Onset Disease in Newborns: ACOG Committee Opinion, Number 782. Obstet Gynecol 2019;134(01):e19-e40 